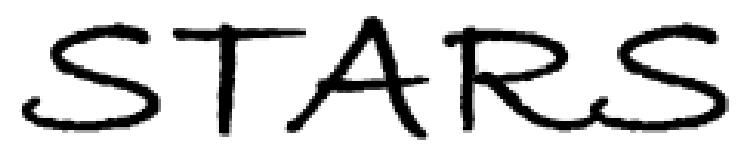

University of Central Florida

STARS

$1-1-2004$

\title{
In-plane switching liquid crystal gel for polarization-independent light switch
}

\author{
Hongwen Ren \\ University of Central Florida \\ Yi-Hsin Lin \\ University of Central Florida \\ Yun-Hsing Fan \\ University of Central Florida \\ Shin-Tson Wu \\ University of Central Florida
}

Find similar works at: https://stars.library.ucf.edu/facultybib2000

University of Central Florida Libraries http://library.ucf.edu

This Article is brought to you for free and open access by the Faculty Bibliography at STARS. It has been accepted for inclusion in Faculty Bibliography 2000s by an authorized administrator of STARS. For more information, please contact STARS@ucf.edu.

\section{Recommended Citation}

Ren, Hongwen; Lin, Yi-Hsin; Fan, Yun-Hsing; and Wu, Shin-Tson, "In-plane switching liquid crystal gel for polarization-independent light switch" (2004). Faculty Bibliography 2000s. 4737.

https://stars.library.ucf.edu/facultybib2000/4737






\section{In-plane switching liquid crystal gel for polarization-independent light switch}

Cite as: J. Appl. Phys. 96, 3609 (2004); https://doi.org/10.1063/1.1789632

Submitted: 03 May 2004 . Accepted: 13 July 2004 . Published Online: 23 September 2004

Hongwen Ren, Yi-Hsin Lin, Yun-Hsing Fan, and Shin-Tson Wu

\section{ARTICLES YOU MAY BE INTERESTED IN}

Fast-switching initially-transparent liquid crystal light shutter with crossed patterned electrodes

AIP Advances 5, 047118 (2015); https://doi.org/10.1063/1.4918277

Electrically induced light scattering from anisotropic gels

Journal of Applied Physics 68, 4406 (1990); https://doi.org/10.1063/1.346190

Anisotropic liquid crystal gels for switchable polarizers and displays

Applied Physics Letters 81, 1432 (2002); https://doi.org/10.1063/1.1502021

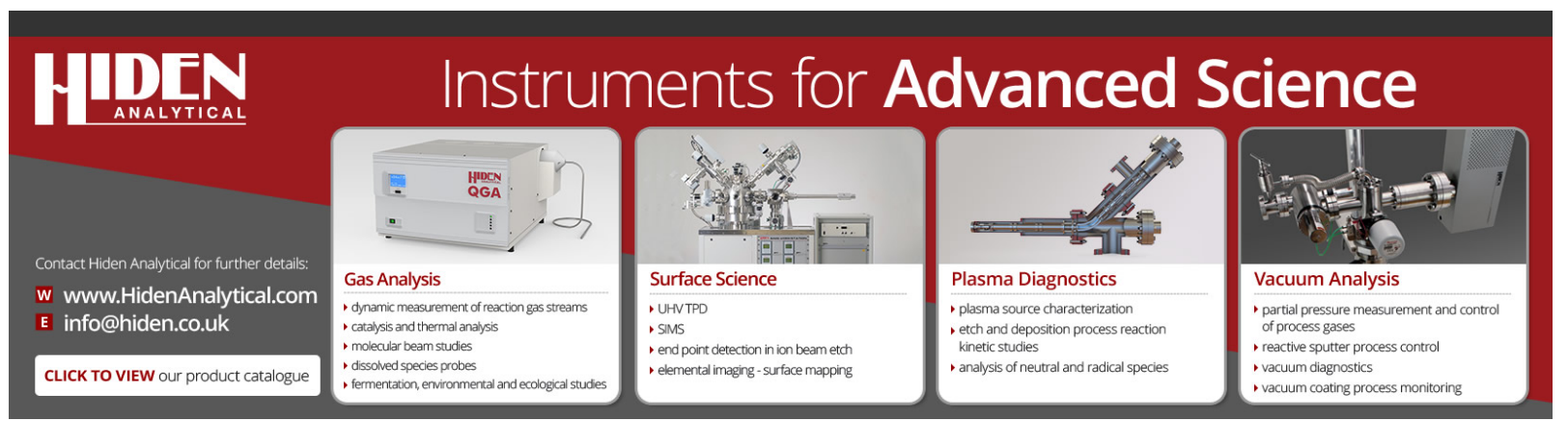




\title{
In-plane switching liquid crystal gel for polarization-independent light switch
}

\author{
Hongwen Ren, Yi-Hsin Lin, Yun-Hsing Fan, and Shin-Tson Wu ${ }^{a)}$ \\ College of Optics and Photonics, University of Central Florida, Orlando, Florida 32816
}

(Received 3 May 2004; accepted 13 July 2004)

\begin{abstract}
An in-plane switching (IPS) liquid crystal (LC) gel for light shutter is demonstrated. In the voltage-off state, the LC gel is transparent. As the voltage exceeds a threshold, light scattering occurs due to microdomain formations. This light scattering is found to be polarization independent. Such an IPS LC gel is a broadband device and has a wide viewing angle and a relatively fast response time. Potential applications for switchable windows, variable optical attenuators, and reflective displays are considered. (C) 2004 American Institue of Physics. [DOI: 10.1063/1.1789632]
\end{abstract}

\section{INTRODUCTION}

Liquid crystal (LC) gels ${ }^{1-3}$ exhibit anisotropic light scattering behavior and have been used as switchable polarizers for sensor protection, telecom optical switches, and reflective displays. In a LC gel, the polymer concentration is usually below $10 \%$. The LC/monomer mixture is injected into a LC cell with surface alignment. Polymerization takes place by photocuring the diacrylate monomer mixed in the LC host. After photopolymerization, the LC molecules are stabilized by the anisotropic cross-linked polymer networks. In the voltage-off state, the LC gel is highly transparent due to ordered molecular alignment. If the applied voltage exceeds a threshold, the polymer networks resist LC molecules from being reoriented by the electric field, resulting in microdomain formation. If the microdomain size is comparable to the incident wavelength, it scatters light. The gel scatters the light whose polarization component is along the cell rubbing direction and transmits the orthogonal component.

Two types of LC gels, homogeneous molecular alignment ${ }^{1-3}$ and homeotropic alignment, ${ }^{4}$ have been developed. By optimizing the curing conditions, ${ }^{5}$ a homogeneous gel exhibits a very high contrast ratio $(>2000: 1)$ but is also polarization dependent. On the other hand, a homeotropic cell is insensitive to polarization, but its contrast ratio is limited to $\sim 10: 1$.

In this paper, we demonstrate a polarization-independent LC gel that uses in-plane switching (IPS) electrodes. ${ }^{6,7}$ In a homogeneous or homeotropic cell, the electrodes are in both substrates and the electric field is in the longitudinal direction. In the IPS cell, the interdigitated electrodes are in only one substrate and the electric field is in the transverse direction. The IPS LC gel exhibits a high contrast ratio, broad bandwidth, wide viewing angle, and fast response time. The device structure and operation mechanisms are described in Sec. II. The experimental details and results are presented in Secs. III and IV, respectively.

\section{DEVICE STRUCTURE}

Figures 1(a) and 1(b) show the electrode and LC mo-

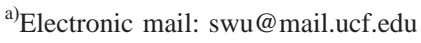

lecular configurations of the IPS LC gel in the voltage-off and voltage-on states, respectively. The IPS substrate consists of interdigitated indium-tin-oxide (ITO) electrodes on the bottom glass substrate. A thin polyimide layer is overcoated on the ITO electrode and buffed along the electrode direction ( $y$ axis). In the voltage-off state, the LC gel is clear due to the homogeneous LC molecular alignment along the $y$ axis. As the applied voltage exceeds the threshold, the horizontal electric field tends to reorient the LC molecules toward the $x$ axis. However, the resisting torque from the cross-linked polymer networks is mainly distributed along the $y$ axis so that the electric field induced LC microdomains do not have a preferred orientation. Thus, the light scattering in the IPS LC gel is independent of polarization. This is different from a homogeneous cell where the electric fieldinduced microdomains are along the rubbing direction so that only the extraordinary ray is scattered.

\section{EXPERIMENTS}

In order to prove the feasibility of these concepts, we prepared an IPS cell with a $20 \mu \mathrm{m}$ electrode width, $100 \mu \mathrm{m}$ electrode gap (the separation between the adjacent electrodes), and $15 \mu \mathrm{m}$ cell gap. In a standard IPS cell used for wide view display, the electrode width is $\sim 5 \mu \mathrm{m}$ and electrode gap is $\sim 10 \mu \mathrm{m}$, in order to lower the operating voltage and improve the contrast ratio. The electrode width and gap are preferably narrow because there is no electric field above


FIG. 1. In-plane switching electrode configuration of the LC gel. LC directors are aligned along the IPS electrodes and stabilized by polymer gel. (a) Transparent state at $V=0$, (b) Scattering state at voltage-on. 


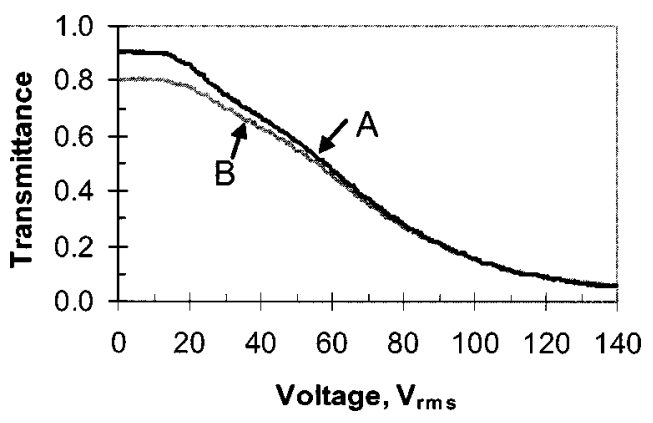

FIG. 2. The voltage dependent transmittance of the IPS LC gel. The polarization of the incident laser beam is $(A)$ perpendicular and $(B)$ parallel to the IPS electrodes. LC=E48, polymer gel $c=3 \%$, cell gap $d=15 \mu \mathrm{m}$, and $\lambda$ $=633 \mathrm{~nm}$.

the center of the electrodes. A large electrode width would lead to a large dead zone which diminishes the device contrast ratio. To fabricate $\mathrm{LC}$ gel, a mixture of $3 \mathrm{wt} \%$ photocurable monomer (bisphenol- $A$-dimethacrylate) ${ }^{8}$ with a small amount of photoinitiator IRG-184 in LC E48 $(\Delta n=0.231)$ host was injected into the IPS cell. The inner surfaces of the IPS cell were coated with a thin polyimide layer and buffed in antiparallel directions along the electrode stripes. The mixture was exposed to UV $(\lambda=365 \mathrm{~nm})$ light at $I$ $\sim 10 \mathrm{~mW} / \mathrm{cm}^{2}$ for $60 \mathrm{~min}$ to form a gel.

\section{RESULTS AND DISCUSSIONS}

The electro-optic properties of the LC gel were studied by measuring the transmittance of the LC gel using an unpolarized He-Ne laser $(\lambda=633 \mathrm{~nm})$. The transmitted light was detected by a photodiode set at $\sim 30 \mathrm{~cm}$ away from the sample, and the corresponding acceptance angle was $\sim \pm 1^{\circ}$. The response time of the $\mathrm{LC}$ gel was recorded by a digital oscilloscope. To examine the polarization effect, a sheet of polarizer was mounted on a rotary stage, which was placed between the laser and the LC gel sample. The voltagedependent transmittance was collected by a LabVIEW data acquisition system. Results are shown in Fig. 2.

Figure 2 shows the measured absolute transmittance of the LC gel at different polarization states. To account for the surface reflections, a similar LC cell filled with E48 LC mixture was used as a reference. The transmittance of the cell is set as $100 \%$. In Fig. 2, curves $A$ and $B$ represent the transmittance for the ordinary (laser polarization perpendicular to the rubbing direction) and extraordinary rays, respectively. In the voltage-off state, curve $A$ has a higher transmittance than $B$. This is because, along the cell rubbing direction, the refractive index of the polymer gel does not match very well with the extraordinary refractive index of the liquid crystal. Thus, a small amount of light is scattered and the LC gel appears slightly hazy. One possible method to reduce the haze is to use a thinner cell gap. In a thinner cell, the LC alignment is more ordered due to the stronger anchoring effects of the alignment layers. The long-range fluctuation in the refractive indices within the gel would be decreased. To maintain high contrast ratio, one should choose high birefringence LC material. From Fig. 2, when the applied voltage increases, the transmittance of $A$ and $B$ declines gradually. In the high voltage range $\left(V>80 V_{\text {rms }}\right)$, curves $A$ and $B$ overlap

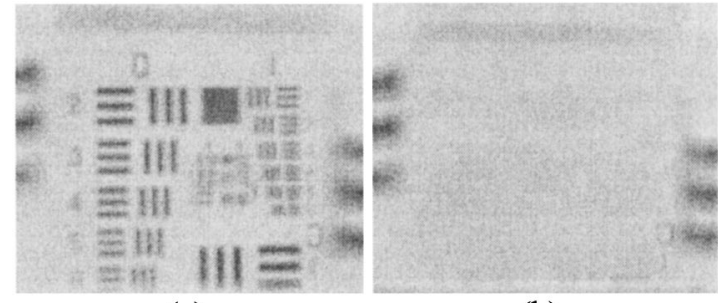

(a)

(b)

FIG. 3. Resolution test of the IPS LC gel as a shutter. The resolution chart is put at $2.5 \mathrm{~cm}$ behind the gel. (a) Transparent state at $V=0$, (b) Scattering state at $140 V_{\text {rms. }}$.

quite well implying that the light scattering is polarization independent. The saturation voltage for the LC gel is $\sim 140 V_{\text {rms }}$ where the contrast ratio reaches $\sim 18: 1$. The high voltage results from the large electrode gap $(100 \mu \mathrm{m})$. The contrast ratio can be further improved if the IPS electrode width is reduced.

The response time of the cell was measured by using a square voltage burst at $f=1 \mathrm{kHz}$ and $\mathrm{V}=140 V_{\text {rms }}$. The turn-on time $\tau_{\text {on }}$ (from transparent to scattering state) and $\tau_{\text {off }}$ (from scattering to transparent state) of the LC gel were measured to be $5 \mathrm{~ms}$ and $16 \mathrm{~ms}$, respectively. The response times of the switchable LC gel can be expressed by ${ }^{2}$

$$
\begin{aligned}
& \tau_{\text {on }}=\tau_{o} /\left[\left(V / V_{\mathrm{th}}\right)^{2}-1\right], \\
& \tau_{\text {off }}=\tau_{o}=\gamma_{1} a^{2} / K \pi^{2}, \\
& V_{\mathrm{th}}=\pi \sqrt{K /\left(\varepsilon_{o} \Delta \varepsilon\right)}
\end{aligned}
$$

where $\gamma_{1}$ is the rotational viscosity of the LC, $\varepsilon_{o}$ is the free space permittivity, $\Delta \varepsilon$ is the low-frequency dielectric anisotropy of the LC, $V$ is the applied voltage, $K$ is the effective elastic constant, and $a$ is the LC domain size. From Eqs. (1) and (2), $\tau_{\text {on }}$ can be reduced by increasing the applied voltage, and $\tau_{\text {off }}$ is determined by the LC parameters and domain size. Increasing polymer concentration would lead to a smaller domain size which, in turn, reduces the response time. The tradeoff is the increased dark state voltage. A lower viscosity LC is always helpful for achieving faster response times.

Similar to a polymer-dispersed liquid crystal ${ }^{9,10}$ and a polymer-stabilized cholesteric texture, ${ }^{11}$ the LC gel is also a broadband device. Figure 3 shows the voltage-off and voltage-on state images of an Air Force resolution chart. The resolution chart was placed at $2.5 \mathrm{~cm}$ behind the LC gel shutter. In the voltage-off state, the gel is relatively transparent as shown in Fig. 3(a). However, its slight haze causes the image to blur which, in turn, degrades the resolution. The high resolution bars are smeared and cannot be observed clearly. In the voltage-on state, the gel scatters light strongly so that the image is effectively blocked, as shown in Fig. 3(b). To reduce haze and then improve resolution, a better refractive index match between the employed LC and polymer could be considered.

Due to polarization independence, the IPS LC gel is attractive for reflective displays. Two important parameters for a reflective display are high contrast ratio and wide viewing angle. The contrast ratio of a reflective mode is the square of 


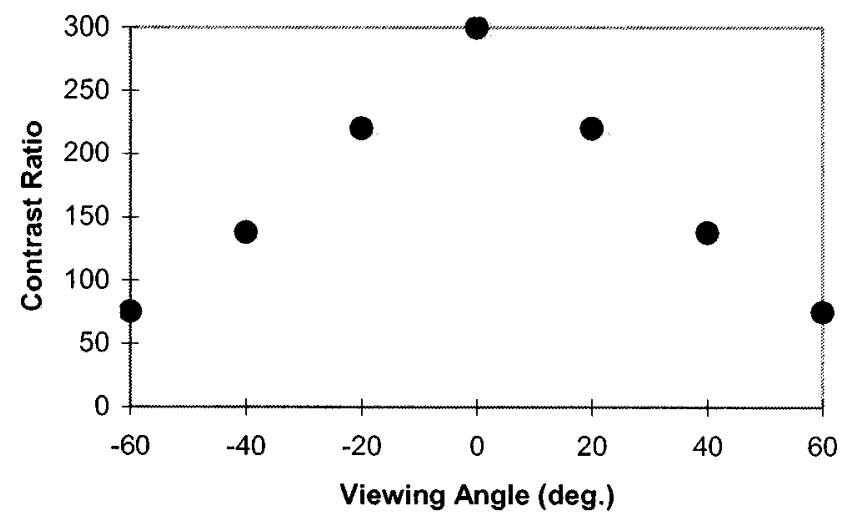

FIG. 4. The measured viewing angle of the IPS LC gel, $\lambda=633 \mathrm{~nm}$.

the corresponding transmissive counterpart. Ideally, in a reflective display the reflector should be embedded in the inner side of the bottom substrate in order to avoid parallax. ${ }^{12}$ In order to demonstrate feasibility, we simply placed a mirror behind the LC gel cell. The He-Ne laser beam passing through the LC gel was reflected by the mirror and detected by the photodetector. The photodetector was placed $\sim 30 \mathrm{~cm}$ behind the LC sample, and the angle between the incident and reflected beams was about $3^{\circ}$. Under this condition, the contrast ratio was measured to be $\sim 300: 1$, which is close to the square of $(\sim 18: 1)$, the contrast ratio of the single pass transmissive cell.

Figure 4 depicts the measured viewing angle of the IPS LC gel using an unpolarized He-Ne laser beam. The viewing angle is wide due to the in-plane switching. From Fig. 4, the contrast ratio remains higher than $\sim 70: 1$ in the $\pm 60^{\circ}$ viewing range.

The high operating voltage of our IPS test cell is due to the large electrode gap. The distance between the adjacent stripes is $100 \mu \mathrm{m}$ and the operating voltage is $140 V_{\text {rms }}$. This corresponds to $1.4 V_{\mathrm{rms}} / \mu \mathrm{m}$. If we use a standard IPS cell which has $\sim 10 \mu \mathrm{m}$ electrode gap the required operating voltage would be reduced to $14 V_{\text {rms }}$.

In our test IPS cell, the width of each finger-shaped electrode is $20 \mu \mathrm{m}$. In the center of the electrode stripes, there is no electric field. The LC gel which covers the electrode stripes cannot be switched. Most of the LC molecules near these regions do not participate in modulating light. Thus, the contrast ratio of our test cell is not as high as expected. To improve the contrast ratio, we should reduce the width of the electrode stripes. Another simple method for improving the contrast ratio is to use a high birefringence LC mixture. High birefringence can be achieved by linearly conjugated molecules. ${ }^{13}$ However, the high birefringence LC compounds are usually associated with a higher viscosity. The isothiocyanato tolane mixtures are found to have $\Delta n>0.4$ while exhibiting a large dipole and low viscosity. ${ }^{14}$ Their potential application in photonics area is foreseeable.

\section{CONCLUSION}

We have demonstrated a polarization independent LC gel for light shutter applications that uses in-plane switching electrodes. Without voltage, the LC gel is clear due to the homogeneous LC alignment. As the applied voltage increases, the LC gel becomes translucent. Such a LC gel is polarization independent and can be obtained with an adequate contrast ratio and a fast switching speed. For reflective displays, the device exhibits a wide viewing angle and high contrast ratio. The electro-optic properties of the LC gel can be improved significantly by reducing the IPS electrode width and electrode gap. Potential applications of IPS LC gel can be found in switchable windows, variable optical attenuators for telecom, and reflective displays.

\section{ACKNOWLEDGMENTS}

The authors would like to thank Hai Jing and Hu Jin from North Liquid Crystal Engineering R\&D Center, Chinese Academy of Sciences for providing the empty IPS cells, and Benjamin $\mathrm{Wu}$ for proofreading the manuscript. This work was supported by DARPA bio-inspired optic synthetic systems program under Contract No. W911NF04C0048.

${ }^{1}$ R. A. M. Hikmet, J. Appl. Phys. 68, 4406 (1990).

${ }^{2}$ R. A. M. Hikmet and H. M. J. Boots, Phys. Rev. E 51, 5824 (1995).

${ }^{3}$ H. Ren and S. T. Wu, Appl. Phys. Lett. 81, 1432 (2002).

${ }^{4}$ R. A. M. Hikmet, Liq. Cryst. 9, 405 (1991).

${ }^{5}$ F. Du and S. T. Wu, Appl. Phys. Lett. 83, 1310 (2003).

${ }^{6}$ R. A. Soref, Appl. Phys. Lett. 22, 165 (1973).

${ }^{7}$ M. Oh-e, M. Yoneya, and K. Kondo, J. Appl. Phys. 82, 528 (1997).

${ }^{8}$ Y. H. Fan, H. Ren, and S. T. Wu, Appl. Phys. Lett. 82, 2946 (2003).

${ }^{9}$ P. S. Drzaic, Liquid Crystal Dispersions (World Scientific, Singapore, 1995).

${ }^{10}$ R. Sutherland, L. V. Natarajan, V. P. Tondiglia, and T. J. Bunning, Chem. Mater. 5, 1533 (1993).

${ }^{11}$ D. K. Yang, L. C. Chen, and J. W. Doane, Appl. Phys. Lett. 60, 3102 (1992).

${ }^{12}$ S. T. Wu and C. S. Wu, Appl. Phys. Lett. 68, 1455 (1996).

${ }^{13}$ S. T. Wu, Phys. Rev. A 33, 1270 (1986).

${ }^{14}$ S. Gauza, H. Wang, C. H. Wen, S. T. Wu, A. J. Seed, and R. Dabrowski, Jpn. J. Appl. Phys., Part 1 42, 3463 (2003). 